\title{
Monte Carlo Algorithm for the Double Exchange Model Optimized for Parallel Computations
}

\author{
Nobuo Furukawa \\ Department of Physics, Aoyama Gakuin University, \\ Setagaya, Tokyo 157-8572, Japan \\ Yukitoshi Motome \\ Institute for Materials Science, Tsukuba University, \\ Tsukuba Japan \\ Hisaho Nakata \\ Scientific and Engineering Research Center, Aoyama Gakuin University, \\ Setagaya, Tokyo 157-8572, Japan
}

\begin{abstract}
A new algorithm for Monte Carlo calculation of the double exchange model is studied. The algorithm is commonly applicable to wide classes of strongly correlated electron systems which involve itinerant electrons coupled with thermodynamically fluctuating fields. Using moment expansions of the density of states with Chebyshev polynomials, the algorithm provides an efficient calculation on large size clusters, especially on parallel computers. Benchmark calculations are performed on Beowulf-type cluster systems with over 100 CPUs in parallel.
\end{abstract}

PACS code: 02.70.Lq 71.10.Fd 75.10.-b 75.70.Pa

Keywords: Double exchange model, Monte Carlo method, moment expansion algorithm, parallel computation 


\section{Introduction}

Double exchange model [1] has been widely studied as a model for itinerant ferromagnetism, which is one of the most interesting subject in the field of strong electron correlations. The model was introduced by Zener in 1951 as a canonical model for perovskite manganites [2], where $e_{\mathrm{g}}$ and $t_{2 \mathrm{~g}}$ electrons of manganese $3 d$ bands are treated as itinerant electrons and localized spins, respectively. Interactions between these two species of electrons are taken into account through on-site Hund's couplings which are stronger than electron hopping energies. The model has been investigated extensively for

half a century, especially in connection with the colossal magnetoresistance phenomena in manganites.

Although the model is simple in its form, thermodynamic properties have not been well known so far. In general, it is difficult to investigate strongly correlated electron systems, both in analytical and numerical methods. Especially in this model, effects of thermal fluctuations around the critical temperature are quite strong so that perturbational approaches as well as mean-field methods do not work in controlled manners.

Recently, dynamical mean-field methods as well as Monte Carlo (MC) calculations on small lattice clusters are performed [1]. These results are more reliable than simple mean-field theories in the sense that they partially take into account the thermal fluctuations. Nevertheless, the former method completely neglects spatial fluctuations while the latter suffers from finite size errors due to the loss of long wavelength fluctuations.

In order to study thermodynamic properties of the model, especially critical phenomena and their influences to electron conductions, it is necessary to perform calculations which properly take into account fluctuation effects. MC calculation on a large size cluster seems to be a promising method, pro- 
vided finite-size extrapolation are treated properly.

In this paper, we study an algorithm for a MC calculation of the double exchange model which improves CPU-time consumption significantly. Using this algorithm, calculations for large size systems become easier, which enables us to perform extrapolations to the thermodynamic limit as well as finite-size scalings.

\section{Monte Carlo algorithm}

The double exchange model is defined by

$$
\mathcal{H}\left(\left\{\vec{S}_{i}\right\}\right)=-t \sum_{<i, j>\sigma} c_{i \sigma}^{\dagger} c_{j \sigma}-J_{\mathrm{H}} \sum_{i} \vec{\sigma}_{i} \cdot \vec{S}_{i}
$$

where $c$ and $c^{\dagger}$ represent operators for itinerant electrons while $\vec{S}$ is the localized classical spins. On-site Hund's coupling $J_{\mathrm{H}}$ gives the interaction energy between itinerant electrons and localized spins. For a given and fixed spin configuration $\left\{\vec{S}_{i}\right\}$, the Hamiltonian is equivalent to a single-body electron system interacting with random magnetic fields. Properties of the system at finite temperatures are obtained through the thermodynamic average over the configuration $\left\{\vec{S}_{i}\right\}$.

Boltzmann weight for a spin configuration $\left\{\vec{S}_{i}\right\}$ is given by

$$
P\left(\left\{\vec{S}_{i}\right\}\right)=\operatorname{Tr} \exp \left[-\beta\left(\mathcal{H}\left(\left\{\vec{S}_{i}\right\}\right)-\mu N\right)\right]=\prod_{m}\left(1+\exp \left[-\beta\left(\varepsilon_{m}-\mu\right)\right]\right)
$$

where Tr represents a grand canonical trace over fermion degrees of freedom. Here, $\varepsilon_{m}$ represent eigenvalues for $\mathcal{H}\left(\left\{\vec{S}_{i}\right\}\right)$. MC sampling of the spin configuration $\left\{\vec{S}_{i}\right\}$ with the probability density $P\left(\left\{\vec{S}_{i}\right\}\right)$ gives stochastical estimates for thermodynamical properties of the system. Direct evaluation of eq. (2) requires all the eigenvalues of $\mathcal{H}\left(\left\{\vec{S}_{i}\right\}\right)$. To obtain all of them 
through a full diagonalization of the Hamiltonian matrix, it is necessary to make a calculation of $O\left(N_{\text {dim }}^{3}\right)$ where $N_{\text {dim }}$ is the Hilbert space dimension of the Hamiltonian.

Alternatively, $P\left(\left\{\vec{S}_{i}\right\}\right)$ can be obtained using the density of states (DOS) [3]. Equation (2) is rewritten as

$$
\log P\left(\left\{\vec{S}_{i}\right\}\right)=\int \mathrm{d} \varepsilon D(\varepsilon) \log (1+\exp [-\beta(\varepsilon-\mu)]),
$$

where $D(\varepsilon)$ is the DOS which depends on the spin configuration $\left\{\vec{S}_{i}\right\}$. Equation (3) is calculated efficiently by the moment expansion algorithm [4, 5]. For a given function $f(\varepsilon)$, we perform a Chebyshev polynomial expansion

$$
\begin{aligned}
& \int \mathrm{d} \varepsilon D(\varepsilon) f(\varepsilon)=\mu_{0} f_{0}+2 \sum_{n \geq 1} \mu_{n} f_{n}, \\
& f_{n}=\int \frac{\mathrm{d} \varepsilon}{\pi \sqrt{1-\varepsilon^{2}}} T_{n}(\varepsilon) f(\varepsilon), \\
& \mu_{n}=\operatorname{Tr} T_{n}(\mathcal{H})=\sum_{\nu}\left\langle\nu\left|T_{n}(\mathcal{H})\right| \nu\right\rangle,
\end{aligned}
$$

where $T_{n}$ is the $n$-th Chebyshev polynomial defined by $T_{0}(\varepsilon)=1, T_{1}(\varepsilon)=\varepsilon$, and $T_{m+1}(\varepsilon)=2 \varepsilon T_{m}(\varepsilon)-T_{m-1}(\varepsilon) .\{|\nu\rangle\}$ is a complete set of kets. We assume here that, in order to ensure the expansion, the Hamiltonian is normalized properly to satisfy $\left|\varepsilon_{m}\right|<1$. Moments for the DOS $\mu_{n}$ are calculated for each given spin configuration $\left\{\vec{S}_{i}\right\}$, while $f_{n}$ are fixed throughout the MC run.

In practice, eq. (6) is calculated as follows. We define

$$
|\nu ; m\rangle \equiv T_{m}(\mathcal{H})|\nu\rangle
$$

Using the recursion formula for the Chebyshev polynomials, we have

$$
|\nu ; m\rangle=2 \mathcal{H}|\nu ; m-1\rangle-|\nu ; m-2\rangle
$$


From the multiplication formula $T_{m+n}=2 T_{m} T_{n}-T_{m-n}$, moments for the DOS are calculated as

$$
\begin{aligned}
\mu_{2 m} & =\sum_{\nu}(\langle\nu ; m \mid \nu ; m\rangle-1) \\
\mu_{2 m+1} & =\sum_{\nu}(\langle\nu ; m \mid \nu ; m+1\rangle-\langle\nu ; 0 \mid \nu ; 1\rangle) .
\end{aligned}
$$

As we see from above, calculation of $P\left(\left\{\vec{S}_{i}\right\}\right)$ based on the moment expansion algorithm costs the CPU time of $O\left(N_{\text {dim }}^{2}\right)$, i.e. the sparse matrix product $\sim O\left(N_{\text {dim }}\right)$ times the trace over the complete set $\sim O\left(N_{\text {dim }}\right)$, provided the electron hopping is short ranged. Therefore, when the lattice size is large, this algorithm provides us a faster calculation than the algorithm based on matrix diagonalization with $O\left(N_{\text {dim }}^{3}\right)$. Moreover, for each $\nu$, eqs. (7)-(10) can be calculated independently. Thus this algorithm is optimized for parallel computations. In contrast, the previous algorithm based on diagonalization of matrices is known to be inefficient for parallelizations.

This algorithm is applicable to wide classes of strongly correlated electron systems where itinerant electrons are coupled with thermally fluctuating fields $\left\{\phi_{i}\right\}$. If one considers $\left\{\phi_{i}\right\}$ as a classical field, thermodynamic averages over $\left\{\phi_{i}\right\}$ are calculated by a MC run. $P\left(\left\{\phi_{i}\right\}\right)$ are calculated by the moment expansion algorithm. It is justified to treat $\left\{\phi_{i}\right\}$ as a classical field at finite temperature, at least near the renormalized classical critical points. It is in general interesting to survey such a region, since the effects of critical fluctuations to the conduction electrons are highly non-trivial.

\section{Benchmark Results and Comments}

Numerical calculations are performed on Aoyama Plus systems [6], Beowulftype clusters of commodity personal computers connected by 100Base-Tx 
Fast Ethernet. Benchmarks are taken on two of the cluster systems, (i) 69 node cluster of dual Pentium II $350 \mathrm{MHz}$ with $384 \mathrm{MB}$ memory (total 138 processors and 26GB memory) and (ii) 11 node cluster of dual Celeron $533 \mathrm{MHz}$ with $256 \mathrm{MB}$ memory (total 22 processors and $2.8 \mathrm{~GB}$ memory). We use MPI for parallel computations. Within a node, shared memory SMP communications based on OpenMP interfaces are used.

\begin{tabular}{ccc}
\hline Lattice size & Previous algorithm & Present algorithm \\
\hline $6 \times 6 \times 6$ & 200 days & 18 hours \\
$8 \times 8 \times 8$ & 16 years & 10 days \\
\hline
\end{tabular}

Table 1: Benchmark result on Pentium II 350MHz computer, for the previous (full diagonalization) algorithm calculated on single node, and the present (moment expansion) algorithm on 64 parallel nodes. CPU time for 10,000 MC steps are shown.

In Table 1 we show the benchmark results for a comparison between the full diagonalization algorithm and the moment expansion algorithm. We take 10,000 MC steps which is typically a minimum number necessary for accurate calculations. Calculations based on the full diagonalization algorithm are performed on one node since it is difficult to make an efficient parallel computation. $64 \mathrm{CPUs}$ are used in parallel for the moment expansion algorithm. As a result, we see a large improvement of the computational speed by the moment expansion algorithm. MC calculation on a $8^{3}$ lattice, which has been virtually impossible by the full diagonalization algorithm, now turns out to be within our reach by the moment expansion algorithm. Using the commodity PC clusters with over 100 CPUs, it is practical to perform a MC calculation for a system with $N_{\operatorname{dim}} \sim 10^{3}$. 
Thus it is now feasible to investigate finite size clusters with systematic series of lattice sizes. Extrapolations to thermodynamic limits as well as finite-size scalings for various thermodynamical properties will be reported elsewhere [7, 8].

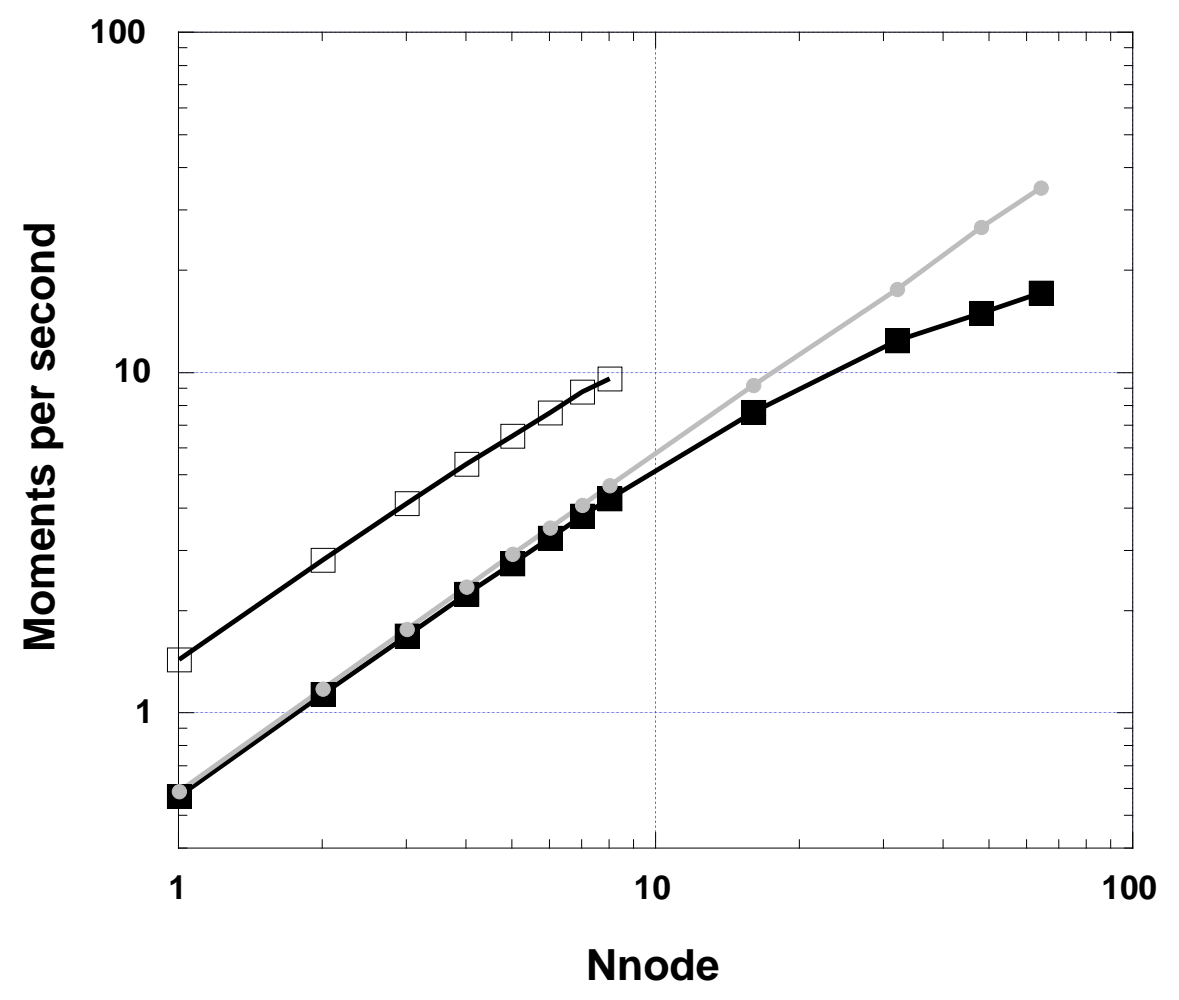

Figure 1: Multiple node benchmark result of the moment expansion algorithm for the double exchange model on a $32 \times 32$ lattice. Calculations are performed on dual PentiumII $350 \mathrm{MHz}$ cluster system (filled square) and dual Celeron 533MHz cluster system (open square) as well as on SGI2800 (filled circle in grey) as a comparison.

Benchmark results for the parallel calculation efficiency are shown in Fig. 1. Computational speeds are indexed by numbers of moments calcu- 
lated per second. We show the results for (i) a PentiumII system, (ii) a Celeron system, as well as (iii) SGI2800 for comparison. We see that in either system the computational speed scales almost linearly with the number of nodes $N_{\text {node }}$. This indicates that the efficiency of the parallel computation is quite high at $N_{\text {node }} \lesssim 10^{2}$. The benchmark result also shows that the program runs equivalently or even faster on commodity personal computer systems compared to the high-performance (and high-cost) parallel computational system.

The moment expansion algorithm is optimized for parallel computations, especially on Beowulf-type commodity systems, in the following sense. (i) The most CPU time consuming part of the MC run is the calculation of $P\left(\left\{\vec{S}_{i}\right\}\right)$, which is completely parallelized. Data transfer occurs only once for each calculation of $P\left(\left\{\vec{S}_{i}\right\}\right)$, while the CPU time for it scales as $O\left(N_{\text {dim }}^{2} / N_{\text {node }}\right)$. Thus, for sufficiently large systems, data transfer time is negligibly small compared to calculation time. High efficiency of the parallel computation is well understood by Amdahl's law. (ii) In general, MC calculations consume small amount of memory. In the present case with $N_{\text {dim }} \sim 10^{3}$, less than $100 \mathrm{kB}$ of memory is used for the vectors $|\nu ; m\rangle$ as well as for the matrix $\mathcal{H}$, which do not overflow from the Level2 (L2) cache of commodity CPUs. Computational speed scales almost linearly with CPU clock speed. (iii) Communications among CPUs as well as access to main memories are, in general, the bottlenecks in the usage of the commodity-type clusters. In our algorithm, these two features conceal the disadvantages.

Since the increase of the CPU clock speed for commodity computers is quite large nowadays, it is now getting easier and easier to construct a commodity computer systems which exhibits a high price-performance for the moment expansion calculations.

Let us finally note that the moment expansion algorithm is applicable 
to many kinds of strongly correlated electron systems. We have obtained a powerful algorithm to investigate thermodynamic properties of various electronic models which have not yet been studied numerically in a systematic way.

\section{Acknowledgements}

The work is supported by the Scientific and Engineering Research Center, Aoyama Gakuin University. MC calculations are performed on Aoyama Plus systems [6] as well as at the Supercomputor Center, ISSP, University of Tokyo.

\section{References}

[1] N. Furukawa, in Physics of Manganites, edited by T. Kaplan and S. Mahanti (Plenum Publishing, New York, 1999), and references therein.

[2] C. Zener: Phys. Rev. 82 (1951) 403.

[3] Y. Motome and N. Furukawa: J. Phys. Soc. Jpn. 68 (1999) 3853.

[4] L. W. Wang: Phys. Rev. B 49 (1994) 10154.

[5] R. N. Silver and H. Röder: Int. J. Mod. Phys. C 5 (1994) 735.

[6] http://www.phys.aoyama.ac.jp/ aaoyama+/index-e.htm]

[7] Y. Motome and N. Furukawa: J. Phys. Soc. Jpn. 69 (2000) 3853.

[8] Y. Motome and N. Furukawa: cond-mat/0103120. 